\title{
Sacred Groves: Myths, Beliefs, and Biodiversity Conservation-A Case Study from Western Himalaya, India
}

\author{
Sushma Singh, ${ }^{1}$ Mudasir Youssouf, ${ }^{2}$ Zubair A. Malik, ${ }^{3}$ and Rainer W. Bussmann ${ }^{4}$ \\ ${ }^{1}$ Department of Environmental Sciences, HNB Garhwal University, Srinagar, Garhwal, Uttarakhand 246174, India \\ ${ }^{2}$ Centre for Environmental Science and Technology, Central University of Punjab, Bathinda, Punjab 151001, India \\ ${ }^{3}$ Department of Botany and Microbiology, HNB Garhwal University, Srinagar, Garhwal, Uttarakhand 246174, India \\ ${ }^{4}$ Saving Knowledge, Casilla 13092, La Paz, Bolivia
}

Correspondence should be addressed to Zubair A. Malik; malikmzubair081@gmail.com

Received 20 July 2017; Accepted 7 September 2017; Published 31 October 2017

Academic Editor: Béla Tóthmérész

Copyright (c) 2017 Sushma Singh et al. This is an open access article distributed under the Creative Commons Attribution License, which permits unrestricted use, distribution, and reproduction in any medium, provided the original work is properly cited.

\begin{abstract}
Religious and traditional beliefs, cultural mores, and practices play a crucial role in the conservation of environment and biodiversity. The present paper describes a case study of two sacred groves in Western Himalaya. Sacred groves (SGs) are patches of land that are communally protected with religious zeal. A preliminary survey was conducted in these SGs to study their role in biodiversity conservation. The data collected included the general information regarding the SGs and the associated deity, nearest human habitation, access to them, and their floral and faunal diversity. Ethnomedicinal property of plants was collected from the indigenous communities. Many taboos are associated with both the SGs, which help in managing resources well through ritual representation. Different festivals are organized, where the local communities reaffirm their commitment to the forest and the deity. Sacred groves, in general, are a valuable tool of biodiversity conservation. But people's changing attitudes, erosion of traditional beliefs, and human impact have caused degradation of sacred groves over the years. Their conservation would not be possible without the active participation of the local people. By improving their living standards and by giving benefits of conservation to them, long-term conservation goals in these SGs can be achieved.
\end{abstract}

\section{Introduction}

Conservation of nature and natural resources has been an important part of cultural ethos, especially in remote rural and indigenous communities in many parts of the world, including India. These communities consider themselves connected with their biophysical environment in a web of spiritual relationship. These rural communities consider specific plants, animals, or even rivers and mountains as their ancestors and protect them. In India, nature worship dates back to the pre-Vedic period (5000 B.C.) and is based on the proposition that all creations of nature have to be protected. The forefathers of these communities were fully aware of the importance and significance of natural resources and the necessity of their conservation for the sustenance of future generations. They lived in harmony with nature and thereby played an important role in conservation of biodiversity [1]. One of the important traditions of nature reverence is to conserve those patches of forest that have been dedicated to a god or goddess or ancestral spirits as "sacred groves." According to Hughes and Chandran [2], sacred groves (SGs) are defined as "segments of landscape containing vegetation, life forms and geographical features, delimited and protected by human societies under the belief that to keep them in a relatively undisturbed state is expression of an important relationship of humans with the divine or with nature." In short, SGs are the relic forest segments preserved in the name of religion and culture. These groves are mostly associated with temples and are also culturally important. They manifest the spiritual and ecological ethos of rural indigenous communities. Various cultural and religious festivals are often arranged by local people within these patches, which they call "Mela." As a way of conservation of nature, SGs have proven to be a well-tried and tested method over thousands of years [3].

Mostly found in Africa and Asia, SGs also exist in Europe and the Americas. Around 100000 to 150000 sacred groves 
TABLE 1: Locations of the studied sacred groves (SGs).

\begin{tabular}{lcc}
\hline Site & Altitude $(\mathrm{m}$ asl $)$ & Geographic coordinates \\
\hline Hariyali Devi (HD) & $1500-2800$ & $\mathrm{~N} 30^{\circ} 19^{\prime} 48.18^{\prime \prime} ; \mathrm{E} 79^{\circ} 00^{\prime} 24.77^{\prime \prime}$ \\
Tungnath (TN) & $3000-4000$ & $\mathrm{~N}^{\prime} 0^{\circ} 29^{\prime} 13.07^{\prime \prime} ; \mathrm{E} 79^{\circ} 13^{\prime} 16.16^{\prime \prime}$ \\
\hline
\end{tabular}

have been reported from India [4]. In India, SGs are especially present in the Himalayan region, Western and Eastern Ghats, Coastal Region, Central Indian Plateau, and Western Desert.

The SGs play an important role in ensuring smooth ecosystem services such as clean environment, that is, air, soil, and water conservation, flora and fauna conservation, carbon sequestration, temperature control, and conservation of traditional knowledge. They are, therefore, of central importance as far as the ecological conservation and policy regarding conservation and management of forest at state and national levels are concerned [5]. Sacred groves serve as a home for birds and mammals, and hence they indirectly help in the conservation of biodiversity [3]. There are several studies carried out by various researchers on this subject, highlighting significant role and potential of the SGs $[1,5$, 6]. The present paper presents a case study of two SGs of Western (Garhwal) Himalaya and the aim of the study was to document (i) the floral and faunal diversity of these SGs and (ii) the myths, beliefs, and taboos related to biodiversity conservation in these SGs.

\section{Materials and Methods}

2.1. Study Area. The present paper describes a case study of two sacred groves from the Western Himalaya. The study was carried out in Uttarakhand, also known as Dev Bhumi (abode or home of gods). Uttarakhand is very rich in biodiversity and there are many SGs for the conservation of this biodiversity. SGs like Chipla Kedar, Tarkeshwar, Hariyali Devi, Binsar, Kot, Kalimath, Goldev, Tapovan, Chandrabadani, Tungnath, and Triyuginarayan are some of the important SGs of Dev Bhumi. The present study was carried out in two SGs, Hariyali Devi and Tungnath, both of which are located in Rudraprayag district (Figure 1 and Table 1).

Hariyali Devi is located above Kodima village at an altitude of $1400 \mathrm{~m}$ in Rudraprayag. The temple contains a bejeweled idol of $M a$ Hariyali Devi astride a lion. The temple houses chiefly three idols, namely, Ma Hariyali Devi, Kshatrapal, and Heet Devi. The temple is open throughout the year but it is more festive at the time of Janmashtami, Navratri, and Deepawali.

Tungnath lies in the upper catchment of the Alaknanda River and the Mandakini River, two major tributaries of the Ganges at an altitude of about 2800-3300 m. Tungnath temple is dedicated to Lord Shiva and is the highest Shiva shrine among the Panch Kedar, $3 \mathrm{~km}$ uphill form Chopta.

2.2. Geology, Soil, and Climate. The rocks of study area are mainly mylonitized gneisses, augen gneisses, schists, and granites constituting Munsiyari Formation [8]. The weathering bedrocks, which provide the bulk of the loose material in these mountains, are crystalline and metamorphic, with sedimentary deposits of Paleozoic age [9]. The soil texture is sandy loam, light grey to brown in color and acidic in nature, with a $\mathrm{pH}$ range between 4 and 5 [10].

Four distinct seasons are observed in the study area: short summer (May-June), Monsoon (July-mid-September), autumn (mid-September-October), and long winter (November-April). The snow cover lasts for about 4-5 months and melts during April-May, which marks the arrival of favorable conditions for plant growth. The growth period lasts for about 5-7 months only [10].

2.3. Sampling Procedures (Methodology). To study the role of the SGs in biodiversity conservation mentioned in Table 1, a preliminary survey was conducted in these areas. Information about these SGs was collected by consulting the elderly people of the villages, governmental and nongovernmental agencies, after receiving prior informed consent, and literature sources (books and scientific journal articles). The data collected included the general information regarding the SGs and the associated deity, nearest human habitation, access to them, and their floral and faunal diversity. Information regarding the ethnomedicinal property of different plants was also collected from the surrounding indigenous communities.

\section{Results and Discussion}

3.1. Biodiversity of Hariyali Devi Sacred Grove. Hariyali Devi SG is rich in floral and faunal diversity (Tables 2, 3, 4, 5, and 6). A total of $80,12,9$, and 7 species of plants, mammals, birds, and butterflies, respectively, were recorded from this SG (Tables 2, 3, 4, 5, and 6). The 80 plant species represented 75 genera and 44 families with different economic values. Rosaceae, with the highest number of species (8), was found to be the dominant family. The number of species varied in different life forms like herbs (37), shrubs (23), and trees (20). Most of the plant species in the SG had one or other ethnomedicinal importance (Tables 2, 3, and 4). The predominant vegetation is represented by Quercus semecarpifolia (kharsu), Quercus leucotrichophora (banj), Rhododendron arboreum (burans), and Lyonia ovalifolia (anyar).

Hariyali Devi forest harbors many sacred animal and butterfly species (Tables 5 and 6). Capricornis sumatraensis, Cervus unicolor, and Felis bengalensis are some common mammalian species. In addition to these, some reptile species were recorded from this SG.

3.2. Biodiversity of Tungnath Sacred Grove. Apart from being a sacred grove, Tungnath is also a part of Kedarnath Wildlife Sanctuary and hence is an important region as far as the conservation of biodiversity is concerned. Tungnath is a home to many rare, threatened, and endangered medicinal 


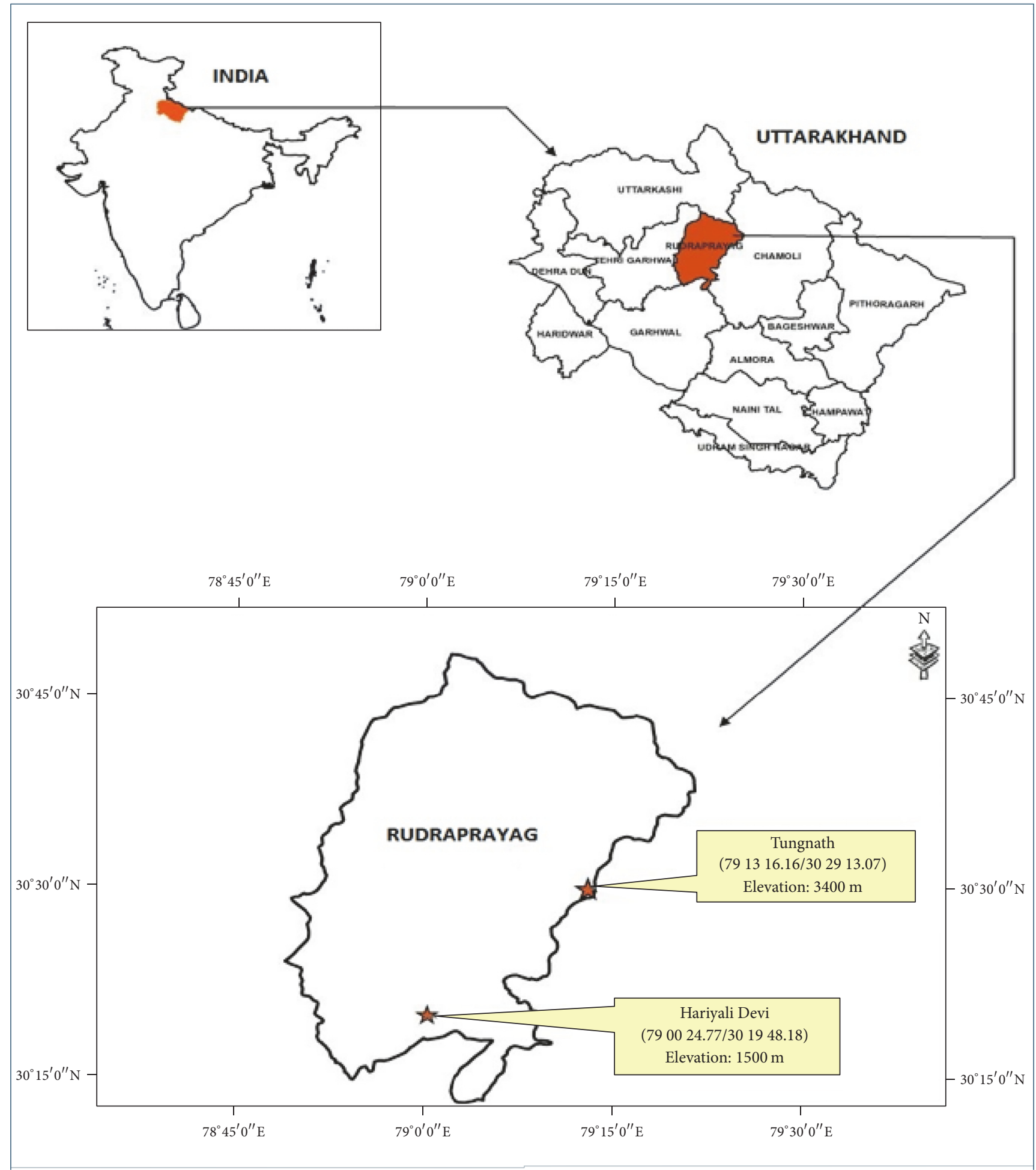

FIGURE 1: Location of the Hariyali Devi and Tungnath sacred groves in Uttarakhand.

plants like Picrorhiza kurroa and Nardostachys jatamansi (Tables 7 and 8). A total of 27 plant species were reported from this SG, which include 8 trees, 10 herbs, and 9 shrubs. Some endangered animals like musk deer, black bear, and so forth are also found there in good numbers (Table 9). Many rare and threatened birds and reptiles are also found there (Table 10).

3.3. Taboos as a Means of Plant and Animal Conservation. Taboos are the unwritten, orally transmitted traditional and 
TABLE 2: List of Tree species (with ethnomedicinal properties) found in Hariyali Devi SG.

\begin{tabular}{|c|c|c|c|}
\hline Botanical name & Vernacular name & Family & Ethnomedicinal property \\
\hline Ilex dipyrena Wall. & Kandara & Aquifoliaceae & $\begin{array}{l}\text { Agriculture implements, fuel, } \\
\text { fodder }\end{array}$ \\
\hline Alnus nepalensis D.Don & Utees & Betulaceae & Fuel, soil binder \\
\hline Betula alnoides Buch.-Ham. ex D.Don & Bhuja patra & Betulaceae & $\begin{array}{l}\text { Wood, fodder, medicine } \\
\quad \text { (rheumatism) }\end{array}$ \\
\hline Benthamidia capitata (Wall.) H. Hara & Bhamora & Cornaceae & Edible (fruit), wood/tiber \\
\hline Cupressus torulosa D.Don ex Lamb. & Surai & Cupressaceae & $\begin{array}{l}\text { Wood, medicine (cough, cold, } \\
\text { and bronchitis) }\end{array}$ \\
\hline Lyonia ovalifolia (Wall.) Drude & Anyar & Ericaceae & $\begin{array}{l}\text { Fuel, medicine (wounds and } \\
\text { boils) }\end{array}$ \\
\hline Rhododendron arboreum $\mathrm{Sm}$. & Burans & Ericaceae & $\begin{array}{c}\text { Fuel, edible flowers, medicinal } \\
\text { (headache, diarrhea, and } \\
\text { dysentery) }\end{array}$ \\
\hline Quercus floribunda Lind. ex A. Camus & Tilonj & Fagaceae & Timber, fodder, fuel \\
\hline Quercus leucotrichophora A. Camus & Banj & Fagaceae & Timber, fodder, fuel \\
\hline Quercus semecarpifolia Sm. & Kharsu & Fagaceae & Fuel/timber and fodder \\
\hline Aesculus indica (Wall. ex Cambess.) Hook. & Pangar & Hippocastanaceae & $\begin{array}{l}\text { Fuel, fodder, medicine } \\
\quad \text { (rheumatism) }\end{array}$ \\
\hline Juglans regia L. & Akhrot & Juglandaceae & $\begin{array}{l}\text { Edible fruit, dye, medicine } \\
\text { (antiseptic, astringent) }\end{array}$ \\
\hline Lindera pulcherrima (Nees) Hook. f. & Cheri & Lauraceae & Wood/fuel, manure \\
\hline Persea gamblei (King ex Hook. f.) Kosterm. & Kauwla & Lauraceae & Agricultural implements/fuel \\
\hline Myrica esculenta Buch.-Ham. ex D.Don & Kaphal & Myricaceae & $\begin{array}{l}\text { Edible fruit, medicine (cough, } \\
\text { fever, and asthma) }\end{array}$ \\
\hline Abies spectabilis (D.Don) Spach. & Morinda & Pinaceae & $\begin{array}{l}\text { Timber/fuel, medicine (fever } \\
\text { and antiseptic) }\end{array}$ \\
\hline Picea smithiana (Wall.) Boiss. & Rai, spruce & Pinaceae & $\begin{array}{c}\text { Wood/timber, medicine (cuts } \\
\text { and sores) }\end{array}$ \\
\hline Pinus wallichiana A. B Jacks. & Kail & Pinaceae & $\begin{array}{l}\text { Fuel, paint, medicine } \\
\text { (antiseptic) }\end{array}$ \\
\hline Prunus cornuta (Wall. ex Royle) Steud. & Jamma & Rosaceae & $\begin{array}{l}\text { Fuel, fodder, medicine } \\
\quad \text { (antipyretic) }\end{array}$ \\
\hline Pyrus pashia Buch-Ham. ex D.Don & Mehal & Rosaceae & $\begin{array}{l}\text { Fuel, fodder, edible, medicine } \\
\text { (diabetes) }\end{array}$ \\
\hline
\end{tabular}

social rules that regulate human behaviour [11]. In rural areas of India like Garhwal (Uttarakhand), there are a number of plants, animals, and even lakes and rivers that are considered sacred and hence no felling or exploitation is being carried out. As a result of this consecration, different species of trees and animals that are economically important or threatened in other areas are preserved and can form a genetic reservoir and serve as a guide against extinction of these species [12]. For example, Dodital and Devariya Tal, two lakes in Uttarkashi and Rudraprayag district of Uttarakhand, are considered sacred, so fishing is completely restricted. There is a taboo that if fishing is done in these lakes, the fisherman will suffer from leprosy [13]. Therefore, the religious belief serves as an instrument of protection of rare and threatened species. Religious beliefs, tradition, and culture are the products of logical internalization of human experience and learning. These practices help in managing resources well through religious or ritual representation.
Trees have a very special role in the ethos of the people in Uttarakhand. Species of trees are worshipped as (1) manifestation of gods, (2) representatives of particular stars and planets, and (3) symbols of the natural elements (energy, water, land, and air), each of which has its own independent and rational meanings [12]. For example, in Garhwal Himalaya, Ficus religiosa is considered to be sacred. There are many instances where communities control the excess use of resource by confining the approach to resources and enforcing compliance through religious belief, rituals, and social convention which at last result in biodiversity conservation in such communities. The roles of religious and cultural beliefs in protecting trees have been observed by other researchers also $[1,12]$. Species such as sacred fig (Ficus religiosa), mountain lion (Felis concolor), and southern pocket gopher (Thomomys umbrinus emotus) are protected by Hindus' taboo all across the Indian subcontinent [13]. In the present study also, we reported some taboos associated 
TABLE 3: List of Shrub species (with ethnomedicinal properties) found in Hariyali Devi SG.

\begin{tabular}{|c|c|c|c|}
\hline Botanical name & Vernacular name & Family & Ethnomedicinal property \\
\hline Hedera nepalensis (K. Koch) & Laguli & Araliaceae & $\begin{array}{l}\text { Medicinal (expectorant, whooping } \\
\text { cough) }\end{array}$ \\
\hline Berberis asiatica Roxb. & Kilmora & Berberidaceae & $\begin{array}{l}\text { Fuel/fodder and medicinal (ophthalmic, } \\
\text { conjunctivitis, and gastritis) }\end{array}$ \\
\hline Sarcococca saligna (D.Don) Mull. Arg. & Piruli & Buxaceae & $\begin{array}{l}\text { Sticks, soil binder, medicinal (joint pains } \\
\text { and fever) }\end{array}$ \\
\hline Abelia triflora R. Br. & Gogti & Caprifolaceae & Walking sticks, fodder \\
\hline Lonicera quinquelocularis Hard. & Taknoi & Caprifolaceae & Edible, walking sticks \\
\hline Viburnum cordifolium Wall. ex DC. & Bhatnoi, guya & Caprifolaceae & $\begin{array}{c}\text { Edible fruits, medicinal (against } \\
\text { menorrhagia-excessive menstruation) }\end{array}$ \\
\hline Elaeagnus parvifolia Wall. ex Royle & Giwain, kanal & Elaeagnaceae & $\begin{array}{l}\text { Medicine (treatment of bloody dysentery, } \\
\text { cardiac tonic, cough, treatment of } \\
\text { afflictions of lungs, cancer treatment), } \\
\text { edible fruits, fodder }\end{array}$ \\
\hline Indigofera heterantha Wall. ex Brandis & Sakina & Fabaceae & $\begin{array}{l}\text { Medicinal (burns, skin diseases, and } \\
\text { ulcers), fodder, edible }\end{array}$ \\
\hline Desmodium elegans DC. & Chamali & Fagaceae & Medicine (antipyretic, vomiting) \\
\hline Deutzia compacta Craib. & Mhujvar & Hydrangeaceae & Medicinal (diuretic) \\
\hline Elsholtzia fruticosa (D.Don) Rehder & Pothi & Lamiaceae & Medicinal (abdominal pain and nausea) \\
\hline Zanthoxylum armatum DC. & Timur & Rutaceae & Medicinal (toothache) \\
\hline Myrsine africana $\mathrm{L}$. & Chupra & Myricaceae & $\begin{array}{l}\text { Medicinal (anthelmintic, antispasmodic, } \\
\text { skin infections) }\end{array}$ \\
\hline $\begin{array}{l}\text { Boenninghausenia albiflora (Hook.) Rchb. ex } \\
\text { Meisn. }\end{array}$ & Pishumar & Rutaceae & $\begin{array}{l}\text { Medicinal (treatment of malaria, } \\
\text { headache, treatment of scabies) }\end{array}$ \\
\hline Rhamnus virgatus Roxb. & Chentuli & Rhamnaceae & Fuel, medicinal (eczema and ringworms) \\
\hline Rosa brunonii Lindl. & Kunja & Rosaceae & $\begin{array}{l}\text { Medicinal (cuts, wounds, and sprains), } \\
\text { soil binder }\end{array}$ \\
\hline Rosa sericea Lindl. & Dhurkunja & Rosaceae & Fodder, edible fruit rich in vitamin $\mathrm{C}$ \\
\hline Rubus foliolosus D.Don & Kala hisar & Rosaceae & $\begin{array}{l}\text { Edible fruits, medicinal (dysentery and } \\
\text { whooping cough) }\end{array}$ \\
\hline Spiraea bella Sims & Kuji & Rosaceae & $\begin{array}{c}\text { Medicinal (wash sores and wounds), } \\
\text { brooms }\end{array}$ \\
\hline Leptodermis lanceolata Wall. & Padera & Rubiaceae & Medicinal (migraines), fodder \\
\hline $\begin{array}{l}\text { Randia tetrasperma (Wall. ex Roxb.) Benth. } \\
\text { \& Hook. f. ex Brandis }\end{array}$ & Kamoli & Rubiaceae & $\begin{array}{l}\text { Fuel, walking sticks, medicinal } \\
\text { (astringent, diuretic, and diarrhea) }\end{array}$ \\
\hline Skimmia anquetilia Tayl. \& Airy Shaw & Nairpatti & Rutaceae & $\begin{array}{l}\text { Agricultural use, sticks, medicinal } \\
\text { (treatment of headache and smallpox) }\end{array}$ \\
\hline Debregeasia longifolia (Burm. F.)Wedd. & Tusara & Urticaceae & $\begin{array}{l}\text { Fodder, used for making ropes, medicine } \\
\text { (treatment of scabies) }\end{array}$ \\
\hline
\end{tabular}

with Hariyali Devi and Tungnath sacred groves. According to villagers, these taboos need to be followed by all. These include the following:

(a) Women are strictly prohibited from entering the sacred forest due to the belief that they are impure.

(b) Fetching/collection of fodder and fuelwood and the movement of women and Shudras (scheduled castes) have been strictly prohibited in this grove since the Mahabharata period. A temple of the goddess Hariyali Devi is located in this forest patch.

(c) Use of tools in any form (knife, sickle, etc.) on the plants and animals will be a step to hurt the sentiments of Devi (goddess). The forest fairies in turn are angered and their wrath can make person mad or deformed and also can lead to disaster in the family of offender.

(d) For a person who starts his journey, if a snake comes across his way, then he has to stop the journey and has to restart only after worshipping the god after an interval of a week.

(e) One week before pilgrimage, the villagers stop eating onion, garlic, egg, and meat.

(f) Anything that is made up of leather is prohibited in the temple and grove. 
TABLE 4: List of Herbaceous species (with ethnomedicinal properties) found in Hariyali Devi SG.

\begin{tabular}{|c|c|c|c|}
\hline Botanical name & Vernacular name & Family & Ethnomedicinal property \\
\hline Barleria cristata $\mathrm{L}$. & Kala bansa & Acanthaceae & $\begin{array}{l}\text { Medicinal (anemia, toothache), soil } \\
\text { binder }\end{array}$ \\
\hline Peristrophe paniculata (Forssk.) Brummitt & Kaknado & Acanthaceae & Medicinal (used against TB) \\
\hline Achyranthes aspera $\mathrm{L}$. & Latjiri & Amaranthaceae & $\begin{array}{l}\text { Medicinal (malarial fever and } \\
\text { muscular sprains) }\end{array}$ \\
\hline Heracleum lanatum Michx. & Kakriya & Apiaceae & Medicinal (nervine and tonic), edible \\
\hline Pimpinella diversifolia DC. & Teroi & Apiaceae & Medicinal (respiratory diseases) \\
\hline Arisaema intermedium Blume & Meen/magmungari & Araceae & Medicinal (burns) \\
\hline Arisaema jacquemontii Blume & Khaprya & Araceae & $\begin{array}{l}\text { Medicinal (antidote of poisonous } \\
\text { mushrooms and snake bite, cough, } \\
\text { kidney, skin diseases) }\end{array}$ \\
\hline Impatiens sulcata Wall. & Chaul & Araliaceae & $\begin{array}{l}\text { Medicinal (antirheumatic and burns), } \\
\text { edible }\end{array}$ \\
\hline Ageratum conyzoides L. & Gundrya & Asteraceae & $\begin{array}{l}\text { Medicinal (anti-inflammatory, } \\
\text { antibacterial) }\end{array}$ \\
\hline Anaphalis triplinervis (Sims) C. B. Clarke & Bugla & Asteraceae & $\begin{array}{l}\text { Medicinal (cuts and wounds, } \\
\text { antiseptic) }\end{array}$ \\
\hline Cynoglossum glochidiatum Wall. ex Benth. & Lichkura & Boraginaceae & $\begin{array}{l}\text { Medicinal (dyspepsia and digestive } \\
\text { disorder), vegetable }\end{array}$ \\
\hline Silene edgeworthii Bocquet & Bakroyla & Caryophyllaceae & Medicinal (eye infections) \\
\hline Stellaria media (L.) Vill. & Badyalu & Caryophyllaceae & $\begin{array}{l}\text { Medicinal (antirheumatic, } \\
\text { anti-inflammatory), vegetable, fodder }\end{array}$ \\
\hline Bryophyllum pinnatum (Lam.) Oken. & Bish-khapura & Crassulaceae & $\begin{array}{l}\text { Medicinal (burns, wounds, and } \\
\text { swellings) }\end{array}$ \\
\hline Dipsacus inermis Wall. & Phulee & Dipsacaceae & $\begin{array}{l}\text { Medicinal (leucoderma and } \\
\text { contusions), edible fruits }\end{array}$ \\
\hline Lathyrus aphaca L. & Kurphail & Fabaceae & Fodder \\
\hline $\begin{array}{l}\text { Swertia angustifolia Buch.-Ham. ex. } \\
\text { D.Don }\end{array}$ & Chirata & Gentianaceae & Medicinal (febrifuge) \\
\hline Geranium nepalense Sweet & Phori & Geraniaceae & $\begin{array}{l}\text { Medicinal (diarrhea, ulcers, and } \\
\text { wounds), tennin }\end{array}$ \\
\hline Hypericum elodeoides Choisy & Basanti & Hypericaceae & $\begin{array}{c}\text { Medicinal (antidepressant, sedative, } \\
\text { rheumatism) }\end{array}$ \\
\hline $\begin{array}{l}\text { Micromeria biflora (Buch.-Ham. ex } \\
\text { D.Don) Benth. }\end{array}$ & Gorakhopan & Lamiaceae & Medicinal (carminative) \\
\hline Origanum vulgare $\mathrm{L}$. & Ban tulsi & Lamiaceae & $\begin{array}{l}\text { Medicinal (antispasmodic, } \\
\text { carminative), vegetable }\end{array}$ \\
\hline Prunella vulgaris $\mathrm{L}$. & Self-heal & Lamiaceae & $\begin{array}{l}\text { Medicinal (wound healing, } \\
\text { expectorant, antiseptic) }\end{array}$ \\
\hline Salvia lanata Roxb. & Ghanyajhar & Lamiaceae & Vegetable and bee-forage source \\
\hline Oxalis corniculata DC. & Bhilmoro & Oxalidaceae & $\begin{array}{c}\text { Medicinal (headache, indigestion, and } \\
\text { diarrhea), vegetable }\end{array}$ \\
\hline Peperomia tetraphylla Hook. \& Arn. & Tirpirya & Piperaceae & $\begin{array}{c}\text { Medicinal (treatment of convulsions, } \\
\text { skin diseases, cough, asthma, kidney } \\
\text { disorders) }\end{array}$ \\
\hline Rumex hastatus D.Don & Almoru & Polygonaceae & Medicinal (astringent) \\
\hline Rumex nepalensis Spreng. & Khatura & Polygonaceae & Medicinal (purgative), vegetable \\
\hline Anemone obtusiloba D.Don & Kanchphool & Ranunculaceae & Medicinal (nervine and sedative) \\
\hline Thalictrum javanicum Blume & Mamiri & Ranunculaceae & $\begin{array}{c}\text { Medicinal (febrifuge, antirheumatic, } \\
\text { and antigout) }\end{array}$ \\
\hline $\begin{array}{l}\text { Fragaria nubicola (Hook. f.) Lindl. ex } \\
\text { Lacaita }\end{array}$ & Gand-kaphal & Rosaceae & Medicinal (earache) \\
\hline Potentilla fulgens Wall. ex Hook. & Bajardantu & Rosaceae & $\begin{array}{c}\text { Medicinal (antidiarrheal, toothache), } \\
\text { edible }\end{array}$ \\
\hline Galium aparine L. & Khuskusa & Rubiaceae & $\begin{array}{c}\text { Medicinal (diuretic and } \\
\text { anti-inflammatory) }\end{array}$ \\
\hline
\end{tabular}


TABLE 4: Continued.

\begin{tabular}{|c|c|c|c|}
\hline Botanical name & Vernacular name & Family & Ethnomedicinal property \\
\hline Solanum erietinum D.Don & Ban-tambakhu & Solanaceae & $\begin{array}{l}\text { Medicinal (vaginal discharges, } \\
\text { inflammation), edible fruits }\end{array}$ \\
\hline Solanum nigrum L. & Banbhatuja & Solanaceae & Medicinal (cough, cold, diuretic) \\
\hline Selinum vaginatum C. B. Clark & Butkeshi & Spigeliaceae & $\begin{array}{c}\text { Medicinal (nervine, sedative, and } \\
\text { analgesic) }\end{array}$ \\
\hline Girardinia diversifolia (Link) Friis & Bhainsya & Urticaceae & $\begin{array}{l}\text { Medicinal (fever, headache, and } \\
\text { swollen joints), fibers, ropes }\end{array}$ \\
\hline Hedychium spicatum Buch.-Ham. ex Sm. & Banhaldi & Zingiberaceae & $\begin{array}{l}\text { Medicinal (analgesic, } \\
\text { anti-inflammatory) }\end{array}$ \\
\hline
\end{tabular}

TABLE 5: List of sacred animal species (with IUCN conservation status) found in Hariyali Devi.

\begin{tabular}{lccc}
\hline Scientific name & Common name & Family & IUCN status* \\
\hline Aonyx cinerea & Asian small-clawed otter & Mustelidae & Vulnerable \\
Capricornis sumatraensis & Serow & Bovidae & Vulnerable \\
Cervus unicolor & Sambar (jado) & Cervidae & Vulnerable \\
Felis bengalensis & Leopard cat & Felidae & Least concern \\
Felis chaus & Jungle cat & Felidae & Least concern \\
Hemitragus jemlahicus & Himalayan tahr & Bovidae & Near threatened \\
Martes flavigula & Himalayan marten (khursyala) & Mustelidae & Least concern \\
Panthera pardus & African leopard & Felidae & Vulnerable \\
Panthera uncia & Leopard (guldar) & Felidae & Endangered \\
Rattus nitidus & Himalayan field rat & Muridae & Least concern \\
Sus scrofa & Wild boar & Suidae & Least concern \\
Ursus arctos & Brown bear & Ursidae & Least concern \\
\hline
\end{tabular}

${ }^{*}$ http://www.iucnredlist.org/search.

TABLE 6: List of birds and butterflies (with IUCN conservation status) found in Hariyali Devi.

\begin{tabular}{lccc}
\hline Scientific name & Common name & Family & \\
\hline & & Birds & IUCN status* \\
Columba eversmanni & Yellow-eyed pigeon & Columbidae & Vulnerable \\
Columba rupestris & Hill pigeon & Columbidae & Least concern \\
Dicrurus macrocercus & Black drongo & Dicruridae & Least concern \\
Gallus gallus & Red junglefowl & Phasianidae & Least concern \\
Streptopelia orientalis & Oriental turtle dove & Columbidae & Least concern \\
Pycnonotus leucogenys & Himalayan bulbul & Pycnonotidae & Least concern \\
Turdoides striata & Jungle babbler & Leiothrichidae & Least concern \\
Dendricitta vagabunda & Rufous treepie & Corvidae & Least concern \\
Urocissa flavirostris & Yellow-billed blue magpie & Corvidae & Least concern \\
\hline & Butterflies & Nymphalidae & Least concern \\
Acraea issoria & Yellow coster & Nymphalidae & Least concern \\
Argynnis $k a m a l a$ & Common silverstripe & Papilionidae & Least concern \\
Delias belladonna horsfieldi & Hill jezebel & Lycaenidae & Least concern \\
Kallima inachus huegelii & Orange oakleaf & Lycaenidae & Least concern \\
Polyura dolon & Stately nawab & Nymphalidae & Least concern \\
Pseudergolis wedah & Tabby & Lycaenidae & Least concern \\
Ypthima sakra & Himalayan five-ring & &
\end{tabular}

\footnotetext{
${ }^{*}$ http://www.iucnredlist.org/search.
} 
TABLE 7: List of Tree species (with ethnomedicinal properties) found in Tungnath SG.

\begin{tabular}{lccc}
\hline Botanical name & Vernacular name & Family & Ethnomedicinal property \\
\hline Acer caesium Wall. ex Brandis & Indian maple & Aceraceae & Fuel, medicinal (for muscular swelling) \\
Ilex dipyrena Wall. & Himalayan holly & Aquifoliaceae & Fuel, fodder, agricultural implements \\
Betula utilis D.Don & Bhojpatra & Betulaceae & Medicinal (diuretic, skin infections) \\
Euonymus tingens Wall. & Spindle tree (kasuree) & Celastraceae & Fuel, also used as dyes \\
Rhododendron arboreum Sm. & Burans & Ericaceae & Fuel, flowers for squash \\
Quercus semecarpifolia Sm. & Kharsu & Fagaceae & Fuel, fodder, and timber \\
Prunus cornuta (Wall. ex Royle) Steud. & Himalayan bird cherry & Rosaceae & Fuel and fodder \\
Taxus wallichiana Zucc. & Himalayan yew & Taxaceae & Medicinal (anticancerous), fuel, timber \\
\hline
\end{tabular}

TABLE 8: List of Herbaceous and Shrub species (with ethnomedicinal properties) found in Tungnath SG.

\begin{tabular}{|c|c|c|c|}
\hline Botanical name & Vernacular name & Family & Ethnomedicinal property \\
\hline \multicolumn{4}{|c|}{ Herbs } \\
\hline Selinum candolle Edgew. & Muur & Apiaceae & Medicinal (analgesic, cough, fever) \\
\hline Silene conoidea $\mathrm{L}$. & Chota takla, thumriya & Caryophyllaceae & $\begin{array}{l}\text { Medicinal (eye infections, } \\
\text { treatment of ophthalmia) }\end{array}$ \\
\hline Morina longifolia Wall. & Kathi, kathoj, sakina & Caprifoliaceae & $\begin{array}{l}\text { Medicinal (wounds and incense, } \\
\text { burns, and boils) }\end{array}$ \\
\hline $\begin{array}{l}\text { Polygonatum verticillatum (L.) } \\
\text { All. }\end{array}$ & Mitha dudhiya & Asparagaceae & $\begin{array}{c}\text { Medicinal (used in treatment of } \\
\text { emaciation, senility, gastric } \\
\text { diseases) }\end{array}$ \\
\hline Corydalis govaniana Wall. & Inderajatta & Papaveraceae & $\begin{array}{c}\text { Medicinal (fever, liver diseases, and } \\
\text { eye infections) }\end{array}$ \\
\hline Cynodon dactylon (L.) Pers. & Dhub & Poaceae & $\begin{array}{l}\text { Medicinal (cuts, wounds, piles, } \\
\text { inflammation, skin diseases) }\end{array}$ \\
\hline $\begin{array}{l}\text { Rubus nepalensis (Hook. f.) } \\
\text { Kuntze }\end{array}$ & Hisar & Rosaceae & Medicinal (cuts and wounds) \\
\hline Picrorhiza kurroa Royle ex Benth & Kutki & Scrophulariaceae & $\begin{array}{c}\text { Medicinal (fever, hepatitis, chronic } \\
\text { dysentery) }\end{array}$ \\
\hline $\begin{array}{l}\text { Nardostachys jatamansi (D.Don.) } \\
\text { DC. }\end{array}$ & Jattamaansi & Valerianaceae & $\begin{array}{l}\text { Medicinal (tranquilizer, sedative, } \\
\text { high blood pressure, used in } \\
\text { dysmenorrhoea for pain relief and } \\
\text { smooth menstrual flow) }\end{array}$ \\
\hline \multicolumn{4}{|c|}{ Shrubs } \\
\hline Berberis aristata DC. & Kingor & Berberidaceae & $\begin{array}{l}\text { Medicinal (used in ophthalmia, } \\
\text { conjunctivitis, ulcers) }\end{array}$ \\
\hline Juniperus indica Bertol. & Guugal & Cupressaceae & Medicinal (cough, cold, and fever) \\
\hline $\begin{array}{l}\text { Rhododendron anthopogon } \\
\text { D.Don }\end{array}$ & Taalisri, burans & Ericaceae & $\begin{array}{c}\text { Medicinal (against respiratory } \\
\text { diseases) }\end{array}$ \\
\hline $\begin{array}{l}\text { Rhododendron campanulatum } \\
\text { D.Don. }\end{array}$ & Chimal/burans & Ericaceae & $\begin{array}{l}\text { Medicinal (chronic rheumatism } \\
\text { and sciatica) }\end{array}$ \\
\hline $\begin{array}{l}\text { Rhododendron lepidotum Wall. } \\
\text { ex. G.Don }\end{array}$ & Burans & Ericaceae & $\begin{array}{l}\text { Medicinal (respiratory and } \\
\text { digestive ailments) }\end{array}$ \\
\hline Clematis buchananiana DC. & Belkangu & Ranunculaceae & $\begin{array}{l}\text { Medicinal (skin ailments, sinus } \\
\text { inflammation, wounds) }\end{array}$ \\
\hline Cotoneaster acuminatus Lindl. & Ruins & Rosaceae & Medicinal (diarrhea and dysentery) \\
\hline $\begin{array}{l}\text { Cotoneaster microphyllus Wall. ex } \\
\text { Lindl. }\end{array}$ & Ruins & Rosaceae & $\begin{array}{c}\text { Anti-inflammatory, cuts, and } \\
\text { wounds }\end{array}$ \\
\hline Leptodermis lanceolata Wall. & Koo-basya & Rubiaceae & Medicinal (migraines) \\
\hline
\end{tabular}


TABLE 9: List of animal species (with IUCN conservation status) found in Tungnath SG.

\begin{tabular}{lccc}
\hline Scientific name & Common name & Family & IUCN status* $^{*}$ \\
\hline Canis aureus & Jackal & Canidae & Least concern \\
Capricornis sumatraensis & Serow & Bovidae & Vulnerable \\
Hemitragus jemlahicus & Himalayan tahr & Bovidae & Near threatened \\
Macaca mulatta & Rhesus macaque & Cercopithecidae & Least concern \\
Moschus chrysogaster & Alpine musk deer & Moschidae & Endangered \\
Ochotona roylei & Himalayan mouse-hare & Ochotonidae & Least concern \\
Panthera pardus & Common leopard & Felidae & Vulnerable \\
Presbytis entellus & Common langur & Cercopithecidae & Least concern \\
Pseudois nayaur & Bharal & Bovidae & Least concern \\
Pteropus giganteus & Indian flying fox & Pteropodidae & Least concern \\
Ursus thibetanus & Himalayan black bear & Ursidae & Vulnerable \\
\hline
\end{tabular}

${ }^{*}$ http://www.iucnredlist.org/search.

TABLE 10: List of birds and reptiles (with IUCN conservation status) found in Tungnath SG.

\begin{tabular}{lccc}
\hline Scientific name & Common name & Family & \\
\hline & Birds & & IUCN status* $^{*}$ \\
Aquila nipalensis & Steppe eagle & Accipitridae & Endangered \\
Gypaetus barbatus & Bearded vulture & Accipitridae & Near threatened \\
Lophophorus impejanus & Himalayan monal & Phasianidae & Least concern \\
Megalaima viridis & White-cheeked barbet & Megalaimidae & Least concern \\
Neophron percnopterus & Egyptian Vulture & Megalaimidae & Least concern \\
Pucrasia macrolopha & Koklass & Phasianidae & Least concern \\
Tragopan melanocephalus & Western tragopan & Phasianidae & Vulnerable \\
Zoothera monticola & Greater long-billed thrush & Turdidae & Least concern \\
\hline & Reptiles & & Agamidae \\
Calotes versicolor & Indian garden lizard & Gekkonidae & Least concern \\
Hemidactylus brookii & Spotted Indian gecko & Elapidae & Near threatened \\
Naja naja & Spectacled cobra & Colubridae & Not evaluated \\
Orthriophis hodgsoni & Himalayan trinket snake & Scincidae & Least concern \\
Scincella himalayanum & Himalayan ground skink &
\end{tabular}

${ }^{*}$ http://www.iucnredlist.org/search.

(g) Killing/hunting of animals and plucking/uprooting of plants are strictly forbidden in the SGs.

3.4. Myths Associated with the Hariyali Devi and Tungnath SGs. According to Hindu Mythology, when Devi Mahamaya was conceived in the form of Devaki's seventh child, the cruel brother of Devaki, Kansa threw Devi Mahamaya aggressively on the ground. Immediately multiple body parts of Devi got scattered all over the earth. The hand fell at Hariyali Devi. Since then, it has become a revered place as Siddha Peeth. The temple is open for all seasons but it is more celebratory at the time of Janmashtami, Navratri, and Deepawali. The myth which prevails according to the Bhagwat Puran is the following: Yogmaya was the sister of Lord Krishna, and she replaced him in the cell of his parents during his birth. When Kansa threw her against the wall, she turned into lightning and came to Hariyali Parvat to make her abode. Since then, the adjoining forest is known as "Hariyali" and is worshipped by people.
The Tungnath temple is the highest Hindu shrine and is believed to be 1000 years old. It has a rich legend linked to the Pandavas, Heroes of Mahabharata epic. According to Hindu Mythology, Vyas Rishi advised the Pandavas that since they were culpable of slaying their own relatives (Kauravas, their cousins) during the Mahabharata war, their act could be pardoned only by Lord Shiva. Consequently, the Pandavas went in search of Shiva who was convinced of the guilt of Pandavas. In order to keep away from them, Shiva took the form of a bull and went into hiding in an underground safe haven of Guptakashi, where Pandavas chased him. But later Shiva's body in the form of bull's body parts rematerialized at five different locations that represent the "Panch Kedar," where Pandavas built temples of Lord Shiva at each location to worship and venerate, seeking his pardon and blessings. Each location is identified with a part of his body; Tungnath is identified as a place where his "Bahu (hands)" were seen. Legend also states that Lord Ram, the chief icon on the Ramayana epic, meditated at the Chandrashila peak, which is close to Tungnath. 


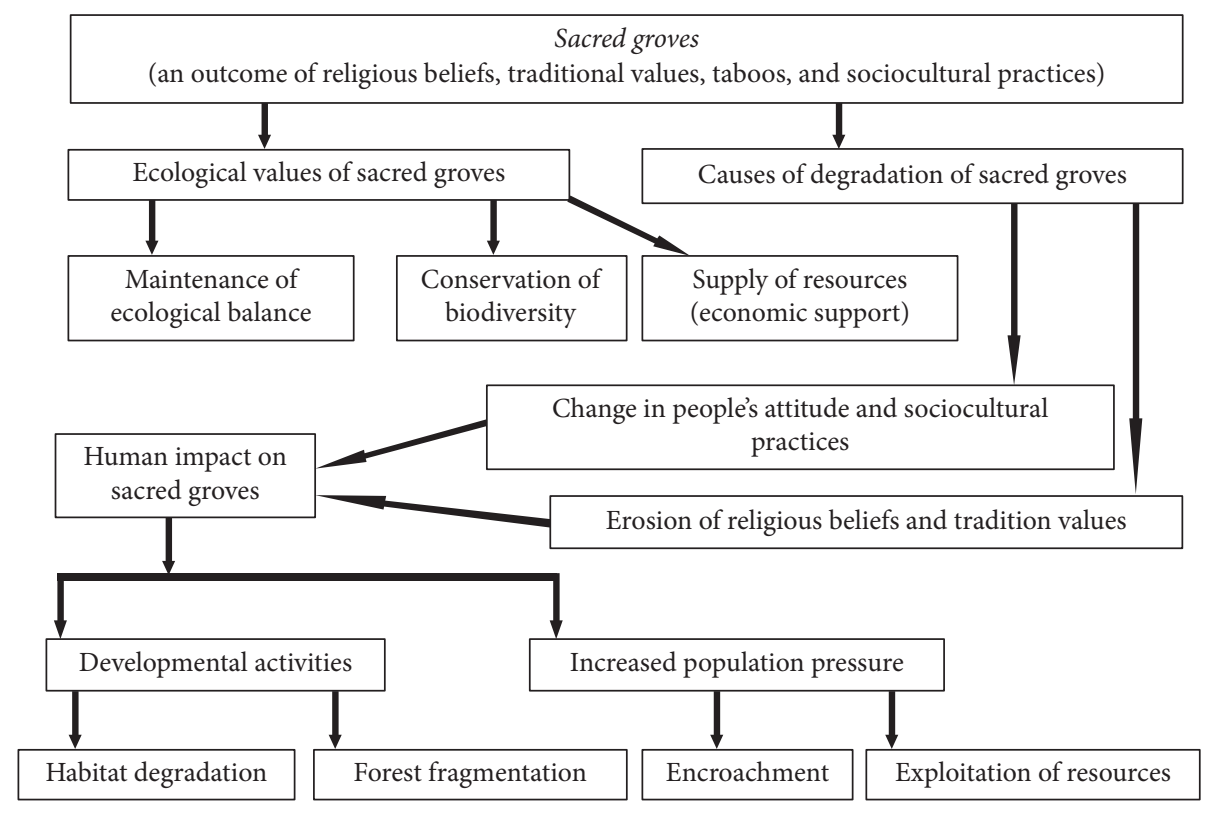

FIGURE 2: Relationships between ecological values, religious beliefs, and traditional values and causes of degradation of sacred groves (courtesy: [7]).

3.5. Festivals (Melas) Associated with the Sacred Groves. SGs are associated with religious rites, festivals, and recreation. The organization and celebration of fairs and festivals have preserved the traditional and sociocultural heritage of Garhwal to a great extent. In Hariyali Devi and Tungnath SGs, festivals (locally called as melas) are organized during April and October every year on the occasion of Navratri, Shivratri, Holi, and so forth. At these melas (festivals), the local communities reaffirm their commitment to the forest and the deity. The heads of the communities supervise the utilization and maintenance of the SGs to ensure that there is no deviation from the village-appointed rules. Anthwal et al. [14] also reported several festivals related to SGs in Uttarakhand. Many plant species have also been associated with religious festivals, namely, Azadirachta indica (Sheela Asthami, Nimb Saptami), Ficus bengalensis (Vat Savitri), Aegle marmelos (Bilvamengal sawan ke somvaar), Musa paradisiaca (Kadii Vrat), and Ficus religiosa (Somvati Amavasya), due to popular and common beliefs [14].

3.6. Conservation of Medicinal Plants. The traditional medical systems of northern India (such as Ayurveda and Tibetan) are a component of culture developed over long time [15]. Medicinally important plants have high importance for religious activities of north Indian native communities that worshiped the plants in the form of god, goddesses, and minor deities [16]. Thus, SGs are the valuable repositories of medicinal and aromatic plants.

Most of the denizens residing in the vicinity of groves are very simple, illiterate, and poor and are almost without any access to modern medicine systems. But they do have conscientiously nourished their traditional knowledge, customs, rituals, and ceremonies with great potency. Local traditional knowledge and the practice of plant-based medicine are still widespread in the rural areas of Garhwal and these play an important role in primary health care [17]. Even the local people prefer to stick to the traditional herbal remedies, and it is due to a situation of having no alternative choices, as well as poverty and belief in the effectiveness of folklore herbal remedies [17]. The denizens living around these SGs have conserved the medicinal plants of these regions for use in a sustainable way by themselves and by their future generations. Religious beliefs and traditional customs have played an important role in this conservation. They have deep faith that if someone from outside the village uproots the medicinal plants from their village, it is treated as an evil act that may bring misery of great order to the village folks.

3.7. Present Status of the HD and TN SGs. Sacred groves, in general, are good instruments of biodiversity conservation. As already mentioned, our ancestors were aware that the natural resources that sustained them should be conserved for the future generations. But, in the course of time, science and technology developed and industries were established and expanded to meet the increasing demands of the people. People's changing attitudes, the erosion of traditional beliefs, and human impact have caused degradation of sacred groves over the years (Figure 2). The same is true for the studied sacred groves. Various anthropogenic activities have altered the structure and function of different ecosystems all over the world [18]. One of the most noticeable effects of ecosystem perturbation has been the depletion of biodiversity [19]. Vanishing of species due to different anthropogenic disturbances like alteration of natural habitats, excessive utilization, pollution, universal climate change, and invasion of nonnative species is so fast that many precious taxa may disappear even before they are documented and identified and their scientific value is discovered [7]. Many scholars have worked on 
conservation of sacred groves through sociocultural practices in different parts of India [1, 12, 20-26].

Lack of awareness in terms of long-term future benefits has resulted in the destruction of SGs. No legislative protection has been implemented so far in India. This has caused considerable ecological damage. Sacred groves have become the victims of deteriorating faith. Such religiously protected areas provide a comprehensive and rich ecological niche as repositories of genetic diversity [12]. The increased threats to SGs can be related to the lack of an in-built conservation effort, higher demands for NTFPs, fuel wood collection, and decrease in the religious faiths along with the reduced commitment of the present generation toward such natural sacred places.

Encroachments of SGs areas by various government departments for different developmental projects, as well as migration and immigration of people, also have contributed to the extinction of SGs. These SGs need to be protected and managed wisely as was done a few decades ago. For providing necessary protection to the SGs and maintaining their natural identity and sanctity, it is imperative that the surrounding population is taken into confidence. The surrounding village communities need to be educated and guided for sustaining the sanctity of existing groves and strengthening them. Conservation of SGs is impossible without the active participation of the local people. Conservation without compensation is only conversation [17, 27]. By improving their living standards and by giving benefits of conservation to them, long-term conservation goals in these SGs can be achieved.

\section{Conclusion}

India has a very high number of scared groves that play an important role in biodiversity conservation because of various myths and religious beliefs associated with them. These SGs have been conserving the biodiversity for many decades. But, nowadays, the attitude of people has changed and this along with the mistrust of traditional beliefs has caused degradation of sacred groves all over India. For improving their degraded condition, it is suggested that the local people living inside and around the SGs need to be taken into confidence, so that long-term conservation goals can be achieved.

\section{Conflicts of Interest}

The authors declare that there are no conflicts of interest regarding the publication of this work.

\section{Acknowledgments}

The authors are thankful to the local people for their cooperation during the study period.

\section{References}

[1] L. S. Kandari, V. K. Bisht, M. Bhardwaj, and A. K. Thakur, "Conservation and management of sacred groves, myths and beliefs of tribal communities: a case study from north-India," Environmental Systems Research, vol. 3, no. 1, 2014.
[2] D. J. Hughes and S. M. D. Chandran, Paper Presented in the Workshop on the Role of Sacred Groves in Conservation and Management of Biological Resources, KFRI, Peechi, India, 1997, Paper presented in the workshop on, the role of sacred groves in conservation and management of biological resources.

[3] A. K. M. N. Islam, M. A. Islam, and A. E. Hoque, "Species composition of sacred groves, their diversity and conservation in Bangladesh," in Conserving the Sacred for Biodiversity Management, P. S. Ramakrishnan, Ed., pp. 163-165, KG Saxena \& UM Chandrashekara, (UNESCO and Oxford-IBH Publishing), New Delhi, India, 1998.

[4] K. C. Malhotra, Y. Gokhale, S. Chatterjee, and S. Srivastava, "Sacred groves in India," in Proceedings of the Aryan Books International, p. 108, New Delhi, India, 2007.

[5] R. Ray and T. V. Ramachandra, "Small sacred groves in local landscape: are they really worthy for conservation?" Current Science, vol. 98, no. 9, pp. 1178-1180, 2010.

[6] H. Singh, T. Husain, and P. Agnihotri, "Haat Kali sacred grove, Central Himalaya, Uttarakhand," Current Science, vol. 98, no. 3, p. 290, 2010.

[7] M. L. Khan, A. D. Khumbongmayum, and R. S. Tripathi, “The sacred groves and their significance in conserving biodiversity an overview," International Journal of Ecology and Environmental Sciences, vol. 34, no. 3, pp. 277-291, 2008.

[8] N. K. Agrawala, Working plan for the Kedarnath Forest Division 1972-73 to 1981-82, Working plan circle, Nainital, Uttar Pradesh, India, 1973.

[9] R. K. Gupta, "Forest types of the Garhwal Himalaya in relation to edaphic and geological formations," Indian Forestor, vol. 4, no. 8, pp. 147-160, 1964.

[10] Z. A. Malik and M. C. Nautiyal, "Species richness and diversity along the altitudinal gradient in Tungnath, the Himalayan benchmark site of HIMADRI," Tropical Plant Research, vol. 3, no. 2, pp. 396-407, 2016.

[11] A. D. Banjo, G. A. Otufale, O. L. Abatan, and E. A. Banjo, “Taboo as a means of plant and animal conservation in South-Western Nigeria: a case study of Ogbe river and its basin," World Applied Sciences Journal, vol. 1, pp. 39-43, 2006.

[12] A. Anthwal, N. Gupta, A. Sharma, S. Anthwal, and K.-H. Kim, "Conserving biodiversity through traditional beliefs in sacred groves in Uttarakhand Himalaya, India," Resources, Conservation \& Recycling, vol. 54, no. 11, pp. 962-971, 2010.

[13] J. Colding and C. Folke, "The relations among threatened species, their protection, and taboos," Ecology and Society, vol. 1, no. $1,1997$.

[14] A. Anthwal, R. C. Sharma, and A. Sharma, "Sacred groves: traditional way of conserving plant diversity in Garhwal Himalaya, Uttaranchal," Journal of American Science, vol. 2, no. 2, pp. 3538, 2006.

[15] C. P. Kala, P. P. Dhyani, and B. S. Sajwan, "Developing the medicinal plants sector in northern India: challenges and opportunities," Journal of Ethnobiology and Ethnomedicine, vol. 2, article 32, 2006.

[16] C. S. Silori and R. Badola, "Medicinal plant cultivation and sustainable development: A case study in the buffer zone of the Nanda Devi biosphere reserve, Western Himalaya, India," Mountain Research and Development, vol. 20, no. 3, pp. 272-279, 2000.

[17] Z. A. Malik, J. A. Bhat, R. Ballabha, R. W. Bussmann, and A. B. Bhatt, "Ethnomedicinal plants traditionally used in health care practices by inhabitants of Western Himalaya," Journal of Ethnopharmacology, vol. 172, article no. 9563, pp. 133-144, 2015. 
[18] Z. A. Malik, R. Pandey, and A. B. Bhatt, "Anthropogenic disturbances and their impact on vegetation in Western Himalaya, India," Journal of Mountain Science, vol. 13, no. 1, pp. 69-82, 2016.

[19] Z. A. Malik, J. A. Bhat, and A. B. Bhatt, "Forest resource use pattern in Kedarnath wildlife sanctuary and its fringe areas (a case study from Western Himalaya, India)," Energy Policy, vol. 67, pp. 138-145, 2014.

[20] B. Sinha and R. K. Maikhuri, "Conservation through sociocultural-religious practice in Garhwal Himalaya: A case study of Hariyali sacred site," in Conserving the Sacred for Biodiversity Management, P. Ramakrishnan, K. G. Saxena, and U. M. Chandrashekhara, Eds., 299, p. 289, Oxford and IBH Publishing, 1998.

[21] S. Sunitha and R. P. Rao, "Sacred groves in Kurnool District, Andhra Pradesh," in Biodiversity, Taxonomy and Conservation of Flowering Plants, M. Sivadasan and P. Mathew, Eds., pp. 367373, Mentor Books, 1999.

[22] R. Basu, "Studies on sacred groves and taboos in Purulia District of West Bengal," Indian Forester, vol. 126, no. 12, pp. 1309-1318, 2000.

[23] C. G. Kushalapa, S. A. Bhagwat, and K. A. Kushalapa, "Conservation and management of sacred groves of Hodagu, Karnataka, South India-a unique approach in," in Tropical Ecosystems: Structure. Diversity and Human Welfare, K. N. Ganeshaiah, U. R. Shaanker, and K. S. Bawa, Eds., pp. 565-569, Oxford IBH Publishing, 2001.

[24] S. A. Bhagwat, C. G. Kushalappa, P. H. Williams, and N. D. Brown, "A landscape approach to biodiversity conservation of sacred groves in the Western Ghats of India," Conservation Biology, vol. 19, no. 6, pp. 1853-1862, 2005.

[25] V. Jaryan, S. K. Uniyal, Gopichand et al., "Role of traditional conservation practice: Highlighting the importance of Shivbari sacred grove in biodiversity conservation," Environmentalist, vol. 30, no. 2, pp. 101-110, 2010.

[26] M. Kala and A. Sharma, "Traditional Indian beliefs: A key toward sustainable living," Environmentalist, vol. 30, no. 1, pp. 85-89, 2010.

[27] Z. A. Malik, Phytosociological behavoiur, anthropogenic disturbances and regeneration status along an altitudinal gradient in Kedarnath Wildlife Sanctuary (KWLS) and its adjoining areas [Ph.D. thesis], HNB Garhwal University, Srinagar Uttarakhand, Uttarakhand, India, 2014. 

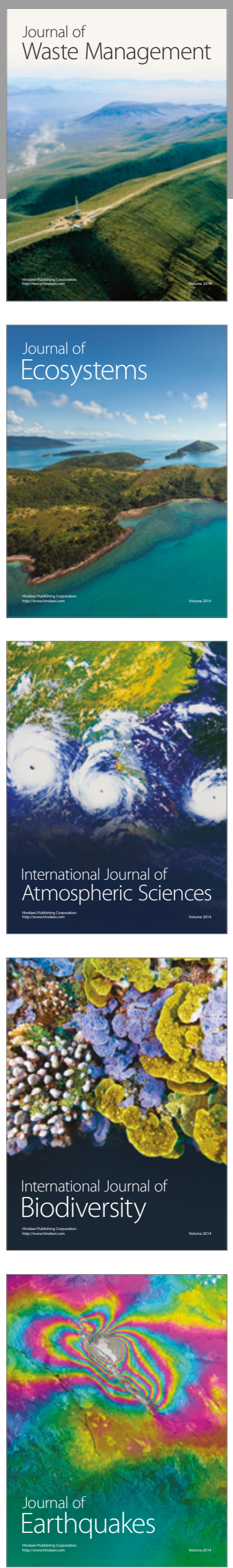
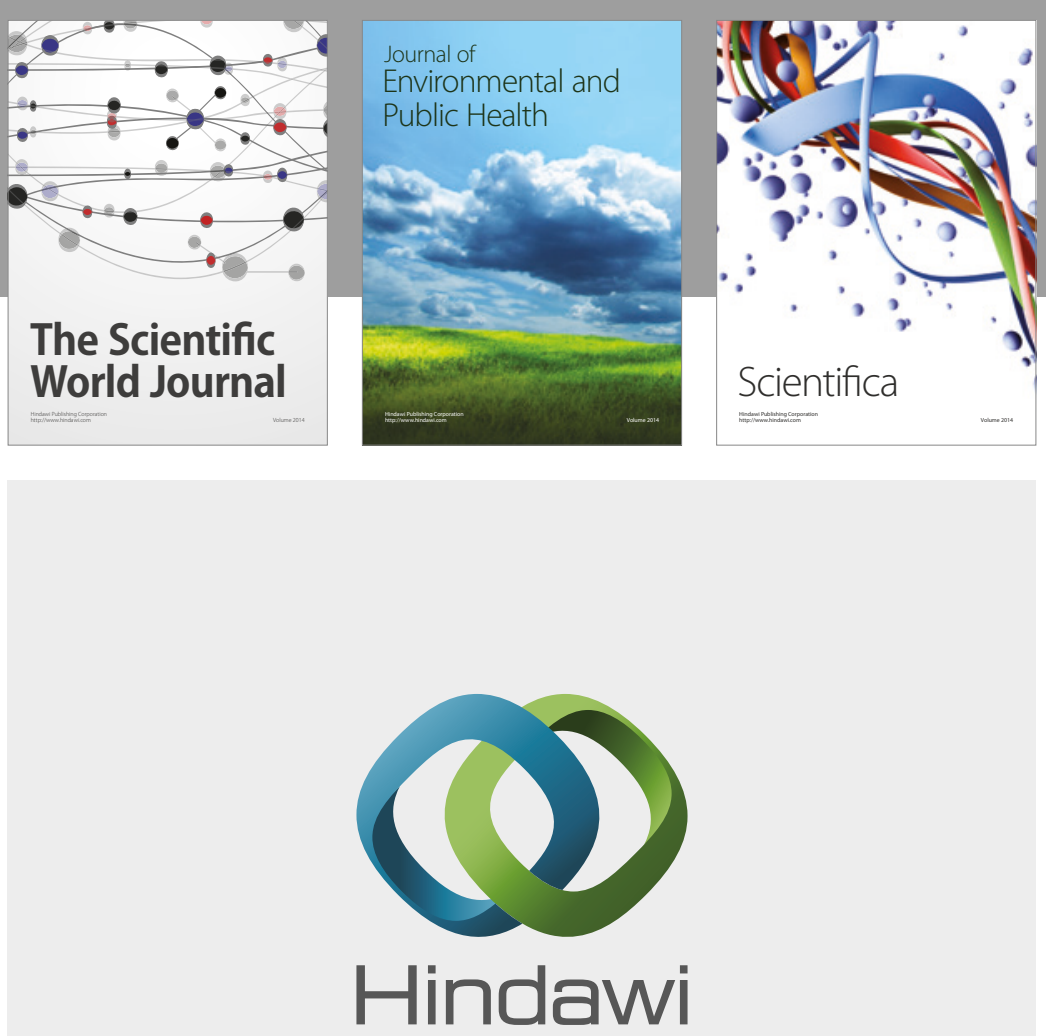

Submit your manuscripts at

https://www.hindawi.com
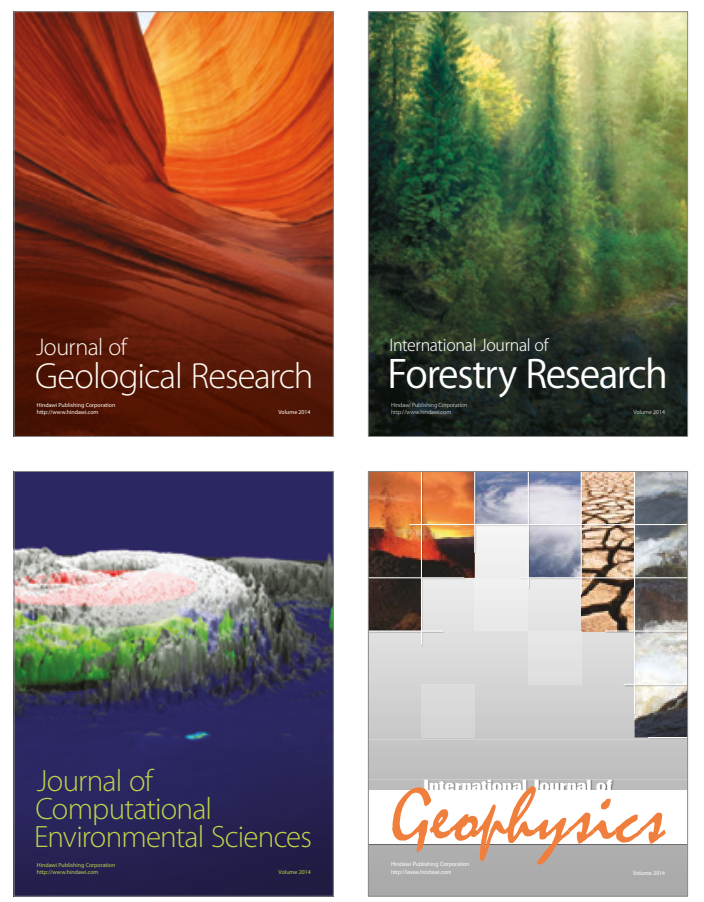
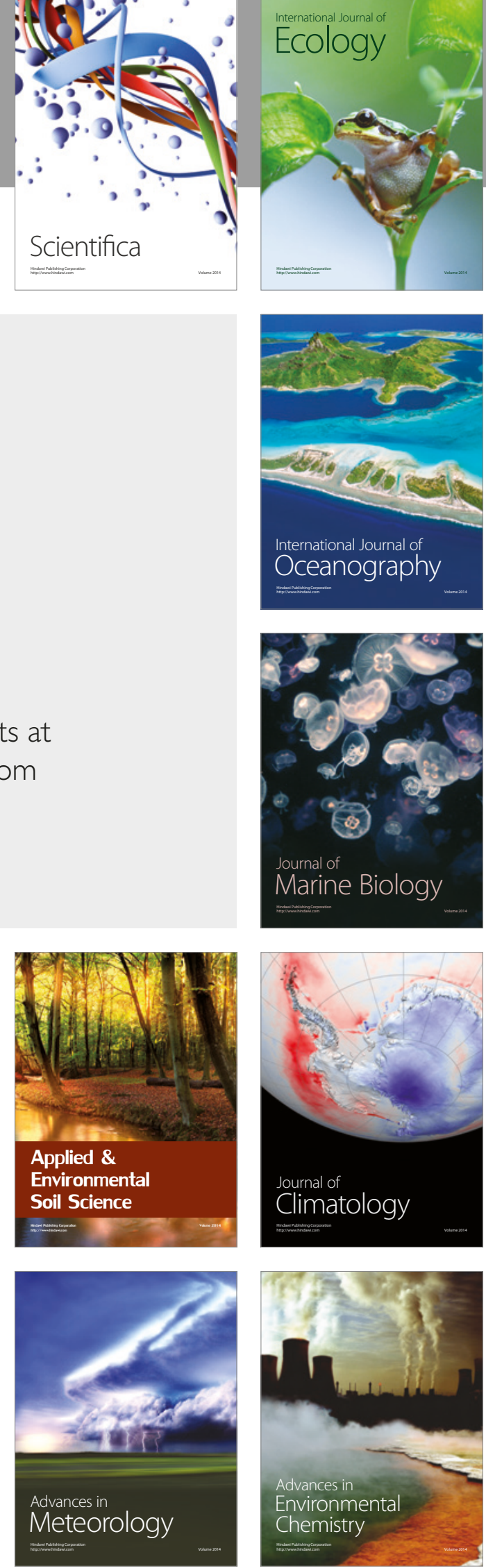